\title{
Erudition of Autism Spectrum Disorder
}

\author{
N. Sharmila Banu \\ Research Scholar \\ Rathinavel Subramaniam College of Arts and \\ Science \\ Sulur, Coimbatore \\ Tamilnadu, India
}

\author{
S. Suganya, PhD \\ Associate Professor \\ Rathinavel Subramaniam College of Arts and \\ Science \\ Sulur, Coimbatore \\ Tamilnadu, India
}

\begin{abstract}
Autism is a developmental disorder characterized by troubles with social interaction and communication and also by restricted and repetitive behavior. Autism affects information processing in the brain by altering nerve cells and their synapses connect and organize. Autism Spectrum Disorder (ASD) is a broad term used to refer to a group of prevalent and debilitating developmental disorders, which includes Autistic Disorder (AD). Autism can look different in different people. It's a developmental disability that affects the way people communicate, behave, or interact with others. There's no single cause for it, and symptoms can be very mild or very severe. It also includes Asperger's Disorder and Pervasive Developmental Disorder-Not Otherwise Specified (PDDNOS).Each of these pervasive neuro-developmental conditions is characterized by impairments in verbal and non verbal communication, social interaction, and a restricted repertoire of activities and interests combined with repetitive behavior and stereotypes. Children with autism are usually diagnosed between the age of 2 and 4 . This paper reveals the main factors involved in ASD, and the diagnosis of this debilitating disorder, causes, symptoms and the treatments which can help to control symptoms.
\end{abstract}

\section{Keywords}

Autism spectrum disorder; Autism; Aspergers Disorder; Pervasive Disorder; Epilepsy

\section{INTRODUCTION}

Autism spectrum disorder was first described by Kanner in 1943 [1] who identified the triad of core characteristics: impaired social interaction and communication involving reduced eye contact, facial expression, and body gestures, a restricted range of interests and repetitive behavior as shown in Figure 1. Autism word comes from the GREEK which means stereotype. Recent data in the United States estimates that 1/68 children received the diagnosis of autism [2].

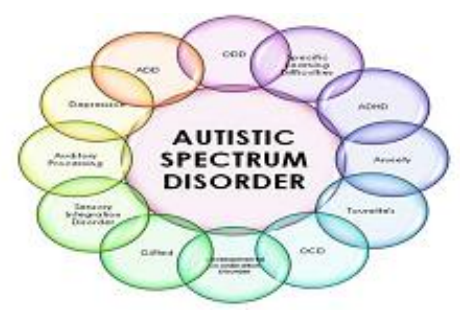

Figure 1: Autism Spectrum Disorder

ASD include the following subtypes: autistic disorder, Asperger syndrome, childhood disintegrative disorder, and pervasive developmental disorder-not otherwise specified (PDD-NOS). Recent data in the United States estimates that
1/68 children received the diagnosis of autism [4]. The Figure 2 represents ASD subtypes.

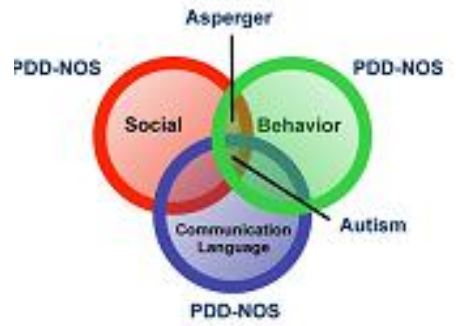

Figure 2: ASD Subtypes

Autistic Disorder is a social situation impairment noted by a failure to exchange non-verbal behaviors such as eye contact, facial expression, body posture, and gestures. Asperger's Syndrome [22] is the impaired ability to utilize social cues such as body language, abstract reasoning, appropriate eye contact, and socialization skills. They tend to have odd behaviors such as being extremely sensitive in responding to stimuli, also exhibiting unusual or repetitive movements. They may exhibit a satisfactory understanding of vocabulary and grammar subjects with a decreased ability to concentrate and or understand humor. Asperger's and Autism together share the issue of recognizing the existence and intentions of others.

The term Pervasive Developmental Disorders is a diagnostic category used to describe many neurological disorders that involve impaired social skills and repetitive behaviors. The PDDs are characterized by their developmental delays in functional and communication skills. They are pervasive, meaning the disease affects many areas. Learning ability is affected but may improve to other levels. They are medical disorders that are not caused by parenting errors, toxins etc. Some cases may be genetic.

\section{CHARACTERISTICS OF ASD}

This is a neurological condition that affects the physical brain and brain chemistry, and can be a severely incapacitating lifelong developmental disability. It affects a variety of bodily functions and may be manifested by few or many symptoms. Even though two children can be diagnosed with the same form of autism, their physiological capabilities may be very different. Many living with ASD will display characteristics that overlap with other disorders in the autism spectrum group. A person with ASD may appear as if they are in their own world, having a unique set of sensory, physical, and mental impairments social situations are difficult, with ASD, as one may present delayed speech with or without meaning, hyperactivity, and repetitive movements. A person with ASD 
may have a time delayed response to learning, or may be incredibly good at other skills. Other traits include having the inability to control emotions, reactions, and behaviors. They may be more sensitive, or they could display a flat facial expression, appearing to be emotionless.

\section{DIAGNOSIS OF AUTISM}

Diagnosing ASD can be difficult because there are no medical or blood tests. The diagnosis is based on behavioral symptoms or features. These features include the absence of or delays in typical developmental milestones and the presence of unusual behaviors. The diagnosis can involve a two-stage process. The first stage is screening, usually by doctors at well-child visits using validated screening checklists that parents fill out. The second stage is a comprehensive diagnostic evaluation usually conducted by a multidisciplinary team that gathers information from an interview and structured observation. The Diagnostic and Statistical Manual of Mental Disorders, Fifth Edition (DSM 5) [5] classified these symptoms into two domains: the social communication and interaction domain, which includes deficits in verbal and nonverbal communication, and the repetitive behavior domain. Parents of children with a diagnosis of autism was generally aware of the problems in their children development by 18 months of age, in the Asperger group concerned emerged later at around 30 months of ages [13]. In order to formally diagnose ASD, children should meet at least three symptoms in the domain of social communication and interaction, and two symptoms in the repetitive behavior domain are shown in (TABLE I) [21].

The symptoms must be present from early childhood and usually limits daily functioning [7]. The National Research Council Committee on Educational Interventions for Children with Autism advised that each child suspected of having ASD have an evaluation that incorporates the following standards: the assessment of multiple areas of functioning including adaptive skills, an appreciation that varies in performance and ability is common in autism, and the use of a developmental perspective when assessing behavior and synthesizing results [17].Epilepsy is one of the complication occurred in the individuals with autism, which means that the messages that travel between nerve cells or neurons becomes scrambled. Because of this, the activity of neurons is disturbed and results in a seizure or loss of consciousness

sIn order to receive a diagnosis for an ASD, the DSM-5requires that individuals meet all three of the criteria in the category of social-communication, impairments, and two of four criteria in the category of restricted and repetitive behaviors [5][6].

\section{SYMPTOMS OF ASD}

Symptoms cause clinically significant impairment in social, occupational, or other important areas of current functioning. The symptoms of ASD are classified into two broad categories: the core and the secondary symptoms [5]. The core symptoms consist of reduced language skills and social interaction, as well as the presence of repetitive and stereotypic behaviors [5][7]. The sign of autism spectrum disorder are shown in (Figure 3)

\begin{tabular}{|c|c|}
\hline Communication & $\begin{array}{l}\text { - Cannot atart or mainain conversaticn } \\
\text { - Communikates with gestures hetosd of words } \\
\text { - Repests words or memoriaed passages }\end{array}$ \\
\hline Social & $\begin{array}{l}\text { - Does not plsy intersctive games } \\
\text { - Avoids eye contact } \\
\text { - Dieploys lsok of empashy }\end{array}$ \\
\hline Sensory & $\begin{array}{l}\text { - Mry find normal noises painful. } \\
\text { hold hands over ears } \\
\text { - Withdraws frem physical conksct } \\
\text { - Rubs surfaces or likis objects }\end{array}$ \\
\hline Play & $\begin{array}{l}\text { - Doesnt imitate actions of others } \\
\text { - Prefers solitary or ritualistic play }\end{array}$ \\
\hline Behaviors & $\begin{array}{l}\text { - Intense tantrums } \\
\text { - Short attention span } \\
\text { - Narrow interests }\end{array}$ \\
\hline
\end{tabular}

Figure 3: Signs of ASD

\section{CAUSES OF ASD}

Many environmental, biological and genetic risk factors have been associated with autism are as follows.

\subsection{Environmental Factors}

There is accumulating evidence supporting a significant contribution of environmental factors to the pathophysiology of ASD [8][9]. Environmental exposure may cause profound changes in brain development and influence neurological processes such as cell differentiation, synapto gene-sis and axon myelination[9] . For instance, it was shown that maternal lifestyle and diet can have beneficial effect on fetal brain development[9]. This should provide accurate information about the potential risks of ASD, and could guide parents and physicians in their decisions.. Environmental factors are also likely to interact with the genetic profile and cause aberrant changes in brain growth, neuronal development, and functional connectivity. Several studies found that tobacco smoking and exposure to alcohol or recreational drugs during pregnancy could cause structural brain anomalies as observed in children with ASD.

There is accumulating evidence supporting a significant contribution of environmental factors to the pathophysiology of ASD [8][9]. Environmental exposure may cause profound changes in brain development and influence neurological processes such as cell differentiation, synapto gene-sis and axon myelination[9] . For instance, it was shown that maternal lifestyle and diet can have beneficial effect on fetal brain development[9]. This should provide accurate information about the potential risks of ASD, and could guide parents and physicians in their decisions.. Environmental factors are also likely to interact with the genetic profile and cause aberrant changes in brain growth, neuronal development, and functional connectivity. Several studies found that tobacco smoking and exposure to alcohol or recreational drugs during pregnancy could cause structural brain anomalies as observed in children with ASD.

\subsection{Biological Factors}

Autism is frequently found to coexist with other diseases or syndromes affecting the central nervous system (CNS), including mental retardation. Turberous sclerosis, neurofibromatosis, epilepsy, congenital rubella, fragile X-syndrome, Rett syndrome, Down syndrome, Williams syndrome, Jubert syndrome (with cerebellar dysfunction), and moebius syndrome are all associated with 
autistic symptoms, suggesting that several different brain areas, perhaps forming a functional unit, could be targeted.

The biological factors that might be involved in ASD are as follows.

- Problems with brain connections

- Problems with growth or overgrowth in certain areas of the brain

- Problems with metabolism (the body's energy production system)

- Problems in the body's immune system, which protects against infections

Neuropathologic and neuroimaging studies have reported developmental anomalies in specific regions of the brain (including the cerebellum, mesial temporal structures, brainstem, basal ganglia, amygdala, frontal and parietal areas and the corpus callosum), although the findings are not fully consistent across studies [12].

\subsection{Genetics}

The identity and number of genes involved remain unknown. The wide phenotypic variability of the ASDs likely reflects the interaction of multiple genes within an individual's genome and the existence of distinct genes and gene combinations among those affected. There are 3 main approaches to identifying genetic loci, chromosomal regions likely to contain relevant genes: 1) whole genome screens, searching for linkage of autism to shared genetic markers in populations of multiplex families (families with $>1$ affected family member); 2) cytogenetic studies that may guide molecular studies by pointing to relevant inherited or denovo chromosomal abnormalities in affected individuals and their families; and 3) evaluation of candidate genes known to affect brain development in these significantly linked regions or, alternatively, linkage of candidate genes selected a priori because of their presumptive contribution to the pathogenesis of autism.

The main syndromes associated with ASD are fragile $\mathrm{X}$ syndrome (FXS) and tuberous sclerosis (TS)[18].FXS is an Xlinked genetic disorder caused by the unstable expansion of a multiple CGG repeat in the FMR1gene[11][18].Its clinical manifestations include epilepsy, learning difficulties, and behavioral problems[14]. The most commonly employed techniques to detect ASD susceptibility genes are the wholeexome sequencing (WES), the chromosomal microarray, and the selective candidate gene analysis[10][18]. WES has been successfully used to identify rare or novel genetic defects in several heterogeneous conditions, including ASD [16].A recent study

found interactive effects between prenatal maternal infection and certain genetic variants on ASD symptomatology [15]. It was shown that children exposed to maternal infection during pregnancy displayed increased rates of social communicative deficits and repetitive/restricted behaviors compared to other children with ASD [15]. Newly described autism susceptibility genes include the neurolignins, neurexins (including CNTNAP 2), human serotonin transporter and human oxytocin receptor genes [20] as well as many others.

The identity and number of genes involved remain unknown. The wide phenotypic variability of the ASDs likely reflects the interaction of multiple genes within an individual's genome and the existence of distinct genes and gene combinations among those affected. There are 3 main approaches to identifying genetic loci, chromosomal regions likely to contain relevant genes: 1) whole genome screens, searching for linkage of autism to shared genetic markers in populations of multiplex families (families with $>1$ affected family member); 2) cytogenetic studies that may guide molecular studies by pointing to relevant inherited or denovo chromosomal abnormalities in affected individuals and their families; and 3) evaluation of candidate genes known to affect brain development in these significantly linked regions or, alternatively, linkage of candidate genes selected a priori because of their presumptive contribution to the pathogenesis of autism.

The main syndromes associated with ASD are fragile X-syndrome (FXS) and tuberous sclerosis (TS)[18].FXS is an X-linked genetic disorder caused by the unstable expansion of a multiple CGG repeat in the FMR1gene[11][18].Its clinical manifestations include epilepsy, learning difficulties, and behavioral problems[14]. The most commonly employed techniques to detect ASD susceptibility genes are the whole-exome sequencing (WES), the chromosomal microarray, and the selective candidate gene analysis[10][18]. WES has been successfully used to identify rare or novel genetic defects in several heterogeneous conditions, including ASD [16].A recent study

found interactive effects between prenatal maternal infection and certain genetic variants on ASD symptomatology [15]. It was shown that children exposed to maternal infection during pregnancy displayed increased rates of social communicative deficits and repetitive/restricted behaviors compared to other children with ASD [15]. Newly described autism susceptibility genes include the neurolignins, neurexins (including CNTNAP 2), human serotonin transporter and human oxytocin receptor genes [20] as well as many others.

\section{SCREENING FOR AUTISM}

Pediatricians play a crucial role in screening for autism spectrum disorders. It is of utmost importance to address parental concerns. The American Academy of Pediatrics recommends developmental screening at every well child visit. For children younger than 24 months, the Infant/Toddler Checklist from the Communication and Symbolic Behavior Scales Developmental Profile may be used [26] [27]. The Modified Checklist for Autism in Toddlers (M-CHAT) is a very useful parental questionnaire for children older than 18 months [26][27] and has been validated in many languages including Arabic, English and French. As soon as the child is found to be at risk, he/she should be immediately referred to a pediatric neurologist, child psychiatrist and a special education/early intervention program [26].

\section{TECHNOLOGY}

During the last decades there have been rapid developments in technology.Several methods in diagnosis and treatment of disabilities are as follows:

- Robotics

- Virtual Reality

- Computer Based Applications

- Mobile based applications

Meta based studies [19] showed that technology based intervention have proven successful in teaching children with autism spectrum disorders. One of the first program to treat children with Autism was TEACCH (Treatment and Education of Autistic and related Communication 
handicapped Children) The uses of technologies for the treatment of ASD can be classified according to their needs, are as follows: (i) the impact of autistic sensory and cognitive impairments on daily life, (ii) cognitive rehabilitation/ remediation that attempt to modify and improve the core deficit in social cognition (iii) special education programs to help children acquiring social and academic skills [24].

\section{TREATMENT FOR AUTISM}

Goals of treatment are to maximize the functional independence and quality of life, minimize the core features of ASD, and alleviate family distress. There is no known cure for autism, although those Asperger syndrome and those who have autism and require little-to-no support are more likely to experience a lessening of symptoms over time. The main goals of treatment are to lessen associated deficits and family distress, and to increase quality of life and functional independence. In general, higher IQs are correlated with greater responsiveness to treatment and improved treatment outcomes.

The type of treatment depends on the individual needs. Because ASD is a spectrum disorder (meaning some children have mild symptoms and others have severe symptoms) and each child who has it is unique, there are a variety of treatments. They can include different kinds of therapies to improve speech and behavior, and sometimes medications to help manage any medical conditions related to autism.Applied Behavior Analysis (ABA) is often used in schools and clinics to help the child to learn positive behaviors and reduce negative ones. This approach can be used to improve a wide range of skills, and there are different types for different situations. Treatment options may include

\subsection{Behavior-Communication Therapies}

Many programs address the range of social, language and behavioral difficulties associated with autism spectrum disorder. Some programs focus on reducing problem behaviors and teaching new skills. Other programs focus on teaching children how to act in social situations or communicate better with others. Applied behavior analysis (ABA) can help children learn new skills and generalize these skills to multiple situations through a reward-based motivation system.

\subsection{Educational Therapies}

Children with autism spectrum disorder often respond to highly structured educational programs. Successful programs typically include a team of specialists and a variety of activities to improve social skills, communication and behavior. Preschool children who receive intensive, individualized behavioral interventions often show good progress.

\subsection{Speech Therapy}

It produces gains in communication skills[25]. It is more effective when the speech therapist collaborates with family, peers, teachers and special educators. It is advised to limit the use of one language at home and at school.

\subsection{Occupational Therapy}

It promotes self-care skills, organization, attention and play skills [25]. Sensory integration remediates deficits in neurological processing of sensory information, thereby improving adaptation of the child to the environment [25].

\subsection{Medications}

No medication can improve the core signs of autism spectrum disorder, but specific medications can help control symptoms.

\section{CONCLUSION}

Autism spectrum disorders are defined by deficits in social and verbal communication and repetitive and stereotyped behaviors not explained by global neurodevelopment delay. The disorder impacts daily life. A genetic study have linked these disorders to known syndromes as well as to recently discovered autism susceptibility genes.ASD is a neurodevelopmental disorder that represents a major public health concern, with pronounced risk for failure to adapt at the social, educational, and psychological level. This complex neurodevelopmental disorder, which is influenced by both genetic and environmenta factors, seems to result from profound changes in brain function and connectivity. Individuals with ASD lack the ability to understand mental states, intentions, thoughts and feelings, irrespective of the emotional state. Early diagnosis and intervention with therapies remains the mainstay of insuring an improved outcomes and a better chance at full integration into society. The study of autism has been very difficult, as there is much variation in the autistic phenotype. Further, many of the abnormalities associated with autism are common to other disorders 


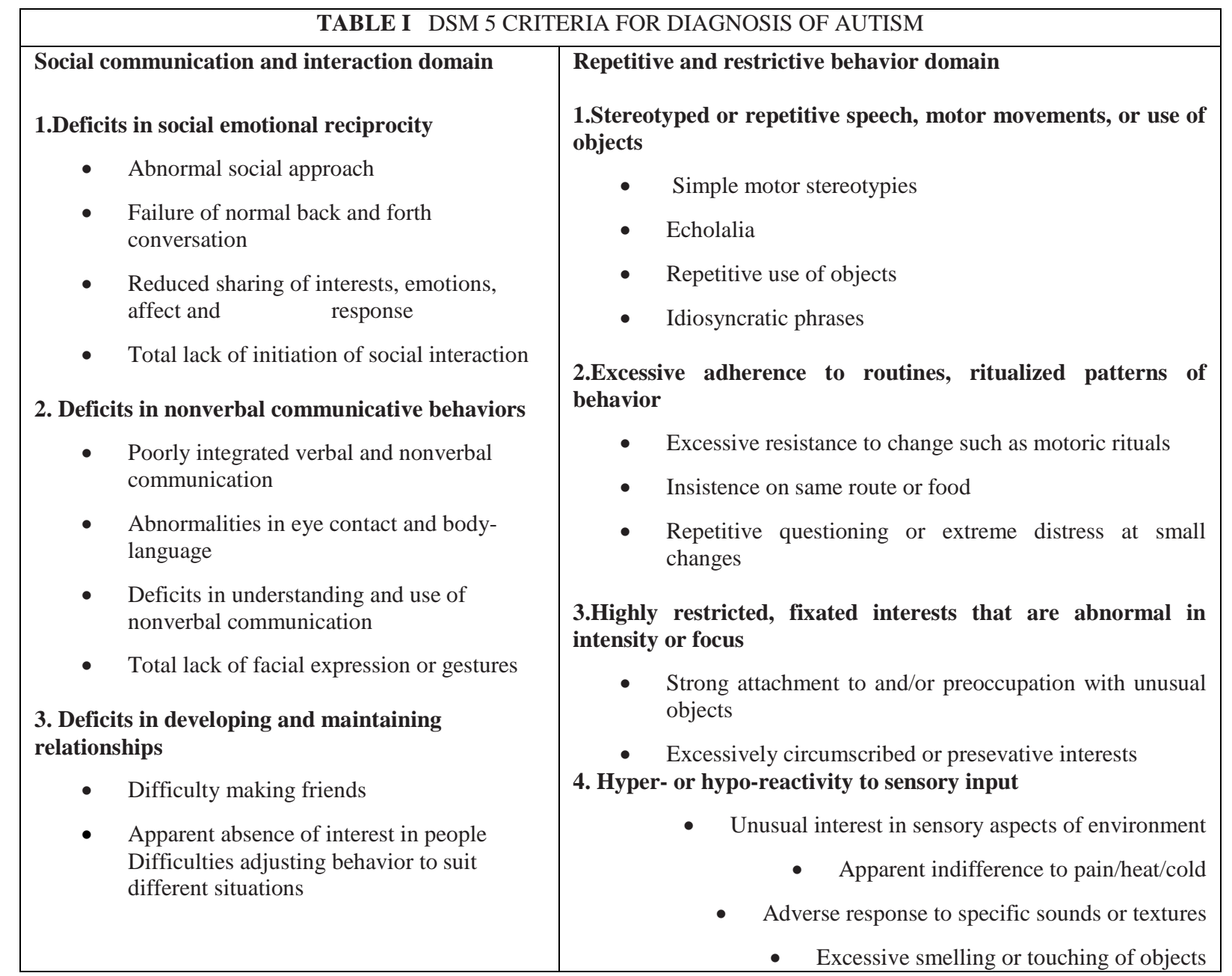

\section{REFERENCES}

[1] Kanner L. "Autistic disturbances of affective contact Acta Paedopsychiatrica" 1968) 35(4):10036

[2] American Psychiatric Association. "Autism Spectrum Disorder. Diagnostic and Statistical Manual of Mental Disorders". Washington, DC: American Psychiatric Association (1994)

[3] Marc Fakhoury, (2015). "Autistic spectrum disorders: A review of clinical features, theories and diagnosis", Int. J.Devl Neuroscience James

[4] Center of Disease Control and Prevention. "Autism.Morbidity and Mortality Weekly Report Surveillance Summaries", March 28, 2014; 63

[5] Barton, M.L., Robins, D.L., Jashar, D., Brennan, L., Fein, D., 2013. "Sensitivity and specificity of proposed DSM-5 criteria for autism spectrum disorder in toddlers". J. Autism Dev. Disord. 43 (5), 1184-1195.

[6] Weitlauf, A.S., Gotham, K.O., Vehorn, A.C., Warren, Z.E., 2014. Brief report: "DSM-5levels of support:a comment on discrepant conceptualizations of severity in ASD" J. Autism Dev. Disorder. 44 (2), 471-476.

[7] Larsson, M., Weiss, B., Janson, S., Sundell,
J., Bornehag, C.G., 2009. "Associations between indoor environmental factors and parental-reported autistic spectrum disorders in children 6-8 years of age". Neurotoxicology 30 (5),822-831.

[8] Lyall, K., Schmidt, R.J., Hertz-Picciotto, I., 2014. "Maternal lifestyle andenvironmental risk factors for autism spectrum disorders". Int. J. Epidemiol. 4343(2), 443-464

[9] Klauck, S.M., 2006. "Genetics of autism spectrum disorder”. Eur. J. Hum. Genet. 14 (6), 714-720.

[10] Muhle, R., Trentacoste, S.V., Rapin, I., 2004. "The genetics of autism". Pediatrics 113 (5)

[11] Penn HE. (2006). "Neurobiological correlates of autism: A review of recent research" . Child Neuropsych, $12: 57-79$.

[12] P. Howlinand A. Asgharian," The diagnosis of autism and Asperger syndrome: findings from a survey of 770 families" Developmental Medicine \& Child Neurology , February 2007

[13] Napolioni, V., Curatolo, P., 2008. "Genetics and molecular biology of tuberoussclerosis complex". Curr. Genomics 9 (7), 475-487.

[14] Mazina, V., Gerdts, J., Trinh, S., Ankenman, K., Ward, T., Dennis, M.Y., 2015." Epigenetics of autism-related impairment: copy number variation 
and maternal infection". J. Dev. Behav. Pediatr. 36 (2), 61-67

[15] Rabbani, B., Tekin, M., Mahdieh, N., 2014.” The promise of whole-exome sequencingin medical genetics". J. Hum. Genet. 59 (1), 5-15

[16] National Research Council Committee on Educational Interventions for Children with Autism. Committee on Early "Intervention for Children with Autism. Goals for Children with Autism and their Families". In: Lord C, McGee J, editors. Educating children with autism spectrum disorders: Report of the Committee on Early Intervention in Autism. Washington, D.C: National Academy of Sciences; 2001. pp. 21-44.

[17] Persico, A.M., Napolioni, V., 2013.“Autism genetics. Behav. Brain Res”. 251, 95-112.

[18] Grynszpan O, Weiss PL, Perez-Diaz F, Gal E. "Innovative technology-based interventions for autism spectrum disorders: a meta-analysis". Autism. 2014;18(4):346-61.

[19] Li X, Zou H, Brown WT." Genes associated with autism spectrum disorder". Brain Research Bulletin 2012; 88:543-52

[20] American Psychiatric Association. "Neurodevelopmental Disorders: Diagnostic and
Statistical Manual of Mental Disorders DSM-5TM", Fifth ed., Arlington, VA: American Psychiatric Association 2013: 50-9

[21] Woodbury-Smith MR, Volkmar FR (January 2009). "Asperger syndrome". Eur Child Adolesc Psychiatry. 18 (1): 2-11

[22] McPartland J, Klin A (2006). "Asperger's syndrome". Adolesc Med Clin. 17 (3): 771-88.

[23] S. Boucenna, A. Narzisi, E. Tilmont, F. Muratori, G.Pioggia, D. Cohen, and M.Chetouani ;“" Interactivetechnologies for autistic children: A review", Springer,2014.

[24] Myers SM, Johnson CP, "The Council on Children with Disabilies. Management of children with autism spectrumdisorders". Pediatrics 2007;120 (5); 1162-82.

[25] Johnson CP, Myers SM. "The Council on Children withDisabilies. Identification and evaluation of children with autism spectrum disorders". Pediatrics 2007 (120) 5; 1183-215

[26] Bradshaw, J.L. and Mattingley, J.B. (1995).Clinical Neuropsychology: Behavioral and Brain Science,San Diego: Academic Press 